\title{
Remarks on Convexity in Dimension $(2,2)$
}

by

\author{
Wojciech KRYŃSKI
}

Presented by Wiesław PLEŚNIAK

Summary. We consider different convexity notions for functions $F: \mathbb{R}^{2 \times 2} \rightarrow \mathbb{R}$. We give a new characterisation of polyconvexity and a sufficient condition for quasiconvexity.

1. Introduction. A continuous function $F: \mathbb{R}^{n \times m} \rightarrow \mathbb{R}$ is quasiconvex if

$$
F(A) \leq \int_{\Omega} F(A+D \varphi(x)) d x
$$

for any matrix $A \in \mathbb{R}^{n \times m}$, and any $\varphi \in C_{0}^{\infty}\left(\Omega, \mathbb{R}^{m}\right)$, where $\Omega \subseteq \mathbb{R}^{n}$ is an open, bounded domain of measure 1. The notion of quasiconvexity was introduced by Morrey [8]. He proved that the lower semicontinuity of the integral functional

$$
I(\varphi)=\int_{\Omega} F(D \varphi(x)) d x
$$

defined for sufficiently regular $\varphi$ is equivalent to the quasiconvexity of $F$. Unfortunately it is hard to verify if a given function is quasiconvex. The following simpler notions were introduced:

1. $F$ is rank-one convex if $F(A) \leq \lambda_{1} F\left(A_{1}\right)+\lambda_{2} F\left(A_{2}\right)$ provided that $\operatorname{rk}\left(A_{1}-A_{2}\right) \leq 1$ and $A=\lambda_{1} A_{1}+\lambda_{2} A_{2}$ is a convex combination, i.e. $\lambda_{1}+\lambda_{2}=1, \lambda_{1}, \lambda_{2} \geq 0$,

2. $F$ is polyconvex if $F(A)=G(T(A))$ for a certain convex (in the usual sense) function $G$, where $T(A)$ is the vector of all determinants of square submatrices of $A$.

It is well known that a polyconvex function is quasiconvex and a quasiconvex function is rank-one convex (see e.g. $[2,6]$ ). In the present paper we will consider the following notion. 
Definition. A function $F: \mathbb{R}^{n \times m} \rightarrow \mathbb{R}$ is $k$-convex if for any convex combination $A=\sum_{i=0}^{k} \lambda_{i} A_{i}$ of matrices $A_{i} \in \mathbb{R}^{n \times m}$ such that $T(A)=$ $\sum_{i=0}^{k} \lambda_{i} T\left(A_{i}\right)$, the following holds:

$$
F(A) \leq \sum_{i=0}^{k} \lambda_{i} F\left(A_{i}\right) .
$$

Note that $\operatorname{rk}\left(A_{1}-A_{2}\right) \leq 1$ iff $T\left(\lambda_{1} A_{1}+\lambda_{2} A_{2}\right)=\lambda_{1} T\left(A_{1}\right)+\lambda_{2} T\left(A_{2}\right)$ and thus a function $F$ is 1-convex iff $F$ is rank-one convex. Obviously, if $F$ is $k$-convex, then it is $l$-convex for any $l<k$.

From now on, we limit ourselves to the case $n=m=2$. It follows from Statement (10) of [4] that $F$ is polyconvex iff it is 5-convex and has a convex lower bound (Theorem 4.4 of [2]). In the present note we will prove that 2 -convexity implies $k$-convexity for any $k$. In particular, we reduce 5 to 2 in Theorem 4.4 of [2].

It follows from our result that quasiconvexity in dimension $2 \times 2$ lies between 2-convexity and 1-convexity. The question whether quasiconvexity is equivalent to rank-one convexity is known as the Morrey conjecture. It is proved to be false in higher dimensions [10] but it is still an open problem in dimension $2 \times 2$ [9]. Polyconvexity is known to be essentially stronger than quasiconvexity (see $[1,3,7,11]$ ), and so is 2-convexity. Recently, a new necessary condition for quasiconvexity has been found [5]. We also refer to [5] for a list of related topics and further references.

2. Results. Let $A \in \mathbb{R}^{2 \times 2}$. We denote by $A^{1}$ and $A^{2}$ the first and second columns of $A$. We write $A=\left[A^{1} A^{2}\right]$.

Lemma 1. Let $A=\sum_{i=0}^{k} \lambda_{i} A_{i}$ be a convex combination with $A_{i} \in \mathbb{R}^{2 \times 2}$. Then $\sum_{i=0}^{k} \lambda_{i} \operatorname{det} A_{i}=\operatorname{det} A$ if and only if $\sum_{i=0}^{k} \sum_{j=0}^{k} \lambda_{i} \lambda_{j} \operatorname{det}\left(A_{i}-A_{j}\right)=0$.

Proof. If $B, C \in \mathbb{R}^{2 \times 2}$, then $\operatorname{det}(B-C)=\operatorname{det} B+\operatorname{det} C-\operatorname{det}\left[B^{1} C^{2}\right]-$ $\operatorname{det}\left[C^{1} B^{2}\right]$. Hence

$$
\begin{aligned}
\operatorname{det} A-\sum_{i=0}^{k} \lambda_{i} \operatorname{det} A_{i} & =\operatorname{det}\left(\sum_{i=0}^{k} \lambda_{i} A_{i}\right)-\sum_{i=0}^{k} \lambda_{i} \operatorname{det} A_{i} \\
& =\sum_{i=0}^{k} \sum_{j=0}^{k} \lambda_{i} \lambda_{j} \operatorname{det}\left[A_{i}^{1} A_{j}^{2}\right]-\sum_{i=0}^{k} \sum_{j=0}^{k} \lambda_{i} \lambda_{j} \operatorname{det} A_{i} \\
& =-\frac{1}{2} \sum_{i=0}^{k} \sum_{j=0}^{k} \lambda_{i} \lambda_{j} \operatorname{det}\left(A_{i}-A_{j}\right),
\end{aligned}
$$

and the lemma follows. 
Remark. Note that, since $\operatorname{det}\left(A_{i}-A_{j}\right)=\operatorname{det}\left(A_{j}-A_{i}\right)$, the condition in the lemma is equivalent to $\sum_{i=0}^{k} \sum_{j=i+1}^{k} \lambda_{i} \lambda_{j} \operatorname{det}\left(A_{i}-A_{j}\right)=0$.

For the sake of convenience we will say that if $A=\sum_{i=0}^{k} \lambda_{i} A_{i}$ and $\operatorname{det} A=\sum_{i=0}^{k} \lambda_{i} \operatorname{det} A_{i}$ are convex combinations then $A$ is a geometric convex combination of the matrices $A_{i}$.

TheOREM 1. If $F$ is 2-convex then $F$ is $k$-convex for any $k$.

Proof. Let $A=\sum_{i=0}^{k} \lambda_{i} A_{i}$ and $\sum_{i=0}^{k} \lambda_{i} \operatorname{det} A_{i}=\operatorname{det} A$. Assume that there are given geometric convex combinations $B_{j}=\sum_{i=0}^{k} \lambda_{i j} A_{i}$, where $j=0, \ldots, n$. If there exist real numbers $\mu_{j} \in[0,1]$ such that $\lambda_{i}=\sum_{j=0}^{n} \mu_{j} \lambda_{i j}$ for any $i=0, \ldots, k$, then $A=\sum_{j=0}^{n} \mu_{j} B_{j}$ is a geometric convex combination of $B_{i}$ :

$$
\operatorname{det} A=\sum_{i=0}^{k} \lambda_{i} \operatorname{det} A_{i}=\sum_{i=0}^{k} \sum_{j=0}^{n} \mu_{j} \lambda_{i j} \operatorname{det} A_{i}=\sum_{j=0}^{n} \mu_{j} \operatorname{det} B_{j} .
$$

Assume that $k>2$. We will prove that there exists a decomposition $A=\sum_{j=0}^{n} \mu_{j} B_{j}$ as above such that $n$ is at most 2 , and moreover, for any fixed $j=0, \ldots, n$ at least one $\lambda_{i j}$ is zero. In other words, each $B_{j}$ will be a convex combination of at most $k$ matrices $A_{i}$. The assumption will imply $F(A) \leq \sum_{j=0}^{n} \mu_{j} F\left(B_{j}\right)$ (since $\left.n \leq 2\right)$ and the inductive procedure will complete the proof.

Set $S_{i}=\sum_{j=0}^{k} \lambda_{i} \lambda_{j} \operatorname{det}\left(A_{i}-A_{j}\right)$. Then $\sum_{i=0}^{k} S_{i}=0$ by Lemma 1 . If there exists $i$ such that $S_{i}=0$, then the Remark implies that

$$
\sum_{j=0, j \neq i}^{k} \sum_{l=j+1, l \neq i}^{k} \lambda_{j} \lambda_{l} \operatorname{det}\left(A_{j}-A_{l}\right)=0,
$$

and one can define $B_{0}=\left(\sum_{j \neq i} \lambda_{j}\right)^{-1} \sum_{j \neq i} \lambda_{j} A_{j}$ and $B_{1}=A_{i}$. In this way we decompose $A$ into the sum of two matrices $A=\left(\sum_{j \neq i} \lambda_{j}\right) B_{0}+\lambda_{i} B_{1}$.

If all $S_{i} \neq 0$ then we may assume that $S_{0}<0$ and $S_{k}>0$ (possibly after permutation of indices). This gives

$$
\sum_{i=1}^{k} \sum_{j=i+1}^{k} \lambda_{i} \lambda_{j} \operatorname{det}\left(A_{i}-A_{j}\right)>0
$$

and

$$
\sum_{i=0}^{k-1} \sum_{j=i+1}^{k-1} \lambda_{i} \lambda_{j} \operatorname{det}\left(A_{i}-A_{j}\right)<0
$$


Let us consider the following convex combinations:

$$
C_{t}=\left((1-t) \lambda_{0}+\sum_{i=1}^{k-1} \lambda_{i}\right)^{-1}\left((1-t) \lambda_{0} A_{0}+\sum_{j=1}^{k-1} \lambda_{j} A_{j}\right)
$$

for $t \in[0,1]$ and

$$
C_{t}=\left((t-1) \lambda_{k}+\sum_{i=1}^{k-1} \lambda_{i}\right)^{-1}\left((t-1) \lambda_{k} A_{k}+\sum_{j=1}^{k-1} \lambda_{j} A_{j}\right)
$$

for $t \in[1,2]$. The Darboux theorem, relations (1), (2) and Lemma 1 imply that there exists $t \in[0,2]$ such that $C_{t}$ is a geometric convex combination of some $A_{i}$. Note that there are always at most $k$ different matrices $A_{i}$ in the sum on the right hand side of the equation which defines $C_{t}$. We set $B_{0}=C_{t}, B_{1}=A_{0}$ and $B_{2}=A_{k}$. This completes the proof.

Corollary 1. A function $F: \mathbb{R}^{2 \times 2} \rightarrow \mathbb{R}$ is polyconvex if and only if it is 2-convex and has a convex lower bound.

COROLlary 2. If $F$ is 2-convex then $F$ is quasiconvex.

Proof. It is known that one can use continuous, piecewise affine functions instead of smooth functions in the definition of quasiconvexity (cf. [2, p. 354]). For such functions the integral is replaced by a sum of the form $\sum_{i=0}^{k} \lambda_{i} F\left(A+A_{i}\right)$, where $k$ is sufficiently large. Moreover, one can see that $A$ is a geometric convex combination of $A+A_{i}$. Thus, if $F$ is $k$-convex for any $k$, then $F$ is quasiconvex. The result follows from Theorem 1.

REMARK. The following problem arises. Find the smallest number $k=$ $k(m, n)$ such that $k$-convexity of a function $F: \mathbb{R}^{n \times m} \rightarrow \mathbb{R}$ implies that $F$ is polyconvex. The natural generalisation of the Morrey conjecture is the question whether quasiconvexity is equivalent to $l$-convexity for some $l>1$ (if $m>2$ ).

\section{References}

[1] J.-J. Alibert and B. Dacorogna, An example of a quasiconvex function that is not polyconvex in two dimensions, Arch. Ration. Mech. Anal. 117 (1992), 155-166.

[2] J. M. Ball, Convexity conditions and existence theorems in nonlinear elasticity, ibid. 63 (1978), 337-403.

[3] - Remarks on the paper 'Basic calculus of variations', Pacific J. Math. 116 (1985), $7-10$.

[4] H. Busemann, G. Ewald and G. C. Shephard, Convex bodies and convexity on Grassmann cones, Math. Ann. 151 (1963), 1-41.

[5] K. Chełmiński and A. Kałamajska, New convexity conditions in the calculus of variations and compensated compactness theory, ESAIM Control Optim. Calc. Var. 12 (2006), 64-92. 
[6] B. Dacorogna, Direct Methods in the Calculus of Variations, Springer, 1988.

[7] T. Iwaniec and J. Kristensen, A construction of quasiconvex functions, Riv. Mat. Univ. Parma (7) 4* (2005), 75-89.

[8] C. B. Morrey, Quasiconvexity and the lower semicontinuity of multiple integrals, Pacific J. Math. 2, 25-53, (1952).

[9] P. Pedregal and V. Šverák, A note on quasiconvexity and rank-one convexity for $2 \times 2$ matrices, J. Convex Anal. 5 (1998), 107-117.

[10] V. Šverák, Rank-one convexity does not imply quasiconvexity, Proc. Roy. Soc. Edinburgh Sect. A 120 (1992), 185-189.

[11] -, Quasiconvex functions with subquadratic growth, Proc. Roy. Soc. London Ser. A 433 (1991), 723-725.

Wojciech Kryński

Institute of Mathematics

Polish Academy of Sciences

Śniadeckich 8

00-956 Warszawa, Poland

E-mail: krynski@mimuw.edu.pl

Received May 1, 2008;

received in final form September 11, 2008 